\title{
Fault Tolerant Control Design of Induction Motor Drive in Electrical Vehicle: A Hybrid Control Approach
}

\author{
M. Boukhnifer ${ }^{1}$, Member IEEE, A. Raisemche ${ }^{1}$ and D. Diallo ${ }^{2}$, Senior Member IEEE \\ ${ }^{1}$ Laboratoire Commande et Systèmes \\ ESTACA, 34 rue Victor Hugo, \\ F-92300 Levallois-Perret. \\ E-mail: moussa.boukhnifer@estaca.fr, aziz.raisemche@estaca.fr \\ ${ }^{2}$ Laboratoire de Génie Electrique de Paris, CNRS UMR 8507, Univ. Paris-Sud \\ 11 rue Joliot Curie Plateau de Moulon, F-91192 Gif-sur-Yvette. \\ E-mail: demba.diallo@1gep.supelec.fr.
}

\begin{abstract}
In this paper, we propose to design a Fault Tolerant Controller (FTC) that can cope both with performance and robustness by the hybridization of two controllers. The distinguished feature of this architecture is that it shows structurally how the controller design for performance and robustness can be done separately. It has the potential to overcome the conflict between performance and robustness of the traditional feedback framework. The controller design works in such a way that the feedback speed control of the induction motor will be solely controlled by the proportional integral $P I$ controller for a nominal model without disturbances and $H_{\infty}$ robust controller will only be activated in the presence of the faults, the uncertainties or external disturbances. This FTC is applied to an induction motor drive for electrical vehicle. The simulation results demonstrate the effectiveness of the proposed hybrid fault tolerant control architecture.
\end{abstract}

Keywords: fault tolerant control, robust control, speed sensor fault, induction motor, electric vehicle.

\section{INTRODUCTION}

Electricity is becoming undoubtfully a more and more important energy source in transportation application like More Electric Aircraft, Hybrid or Electric Vehicle (EV). In fact besides the secondary auxiliaries that have been for a long time using electricity, primary auxiliairies such as front wheel or landgear for aircraft or powertrain in EV are powered with electricity. However in these applications, the security and safety levels are very high. The great amount of experience in the design and maintenance of mechanical systems for more than a century has raised the safety and security levels. This is the challenge faced by electrical engineering. Particularly at fault occurence in the electrical energy conversion chain, the control must ensure that the fault is mitigated other by redundancy or reconfiguration of the parameters or the structure. This is the task devoted to FTC.

Numerous FTC control strategies have already been implemented in the literature. A FTC approach for a fourwheel independently-driven EV has been proposed in [?] . An adaptive control based on passive fault-tolerant controller is designed to ensure the vehicle system stability and to track the desired vehicle motion at fault occurence. Simulations results are given using CarSim. In [?] two techniques for maintaining a constant torque at fault occurence are applied to two different aerospace drives.

Moreover, several works have been reported of fault tolerant control applied to mechatronic systems [?], six-phase induction machine [?], hydraulic actuator [?], vehicle [?] dynamics, spacecraft system [?]. Several works have also been reported on the modeling and the design of the electric vehicle [?] [?].

This paper proposes to combine both fault tolerant and robust approaches to control the speed of an induction motor drive dedicated to propel an electrical vehicle.

The distinguished feature of the proposed controller architecture is that it shows structurally how the controller design for performance and robustness can be done separately. It has the potential to overcome the conflict between performance and robustness in the traditional feedback framework. When a sensor fails or degrades, the controller switches and uses the observer's output (as a software redundancy) instead of the original system's output. The controller architecture works in such a way that the feedback control system will be solely controlled by the proportional integral $P I$ performance controller for a nominal model and the $H_{\infty}$ robust controller will only be activated in the presence of the faults, the uncertainties or external disturbances.

This paper is organised as follows. Section 2 presents the induction motor drive model. We present the proposed FTC control architecture in section 3. Section 4 presents the simulation results of the proposed hybrid FTC controller in case of external perturbations or faulty sensor operation. Finally a conclusion closes the paper.

\section{INDUCTION MOTOR DRIVE MODELING}

\section{A. Induction Motor State Space Model}

To model the induction motor of the electrical vehicle given by the Fig. ??, we use the usual assumptions and Park transformation. The model is described in the $(d, q)$ reference frame related to the rotating field. The stator currents $\left(I_{d s}, I_{q s}\right)$, the rotor fluxes $\left(\phi_{d r}, \phi_{q r}\right)$ and the rotation speed $\omega_{r}$ are considered as state variables. The derived model 


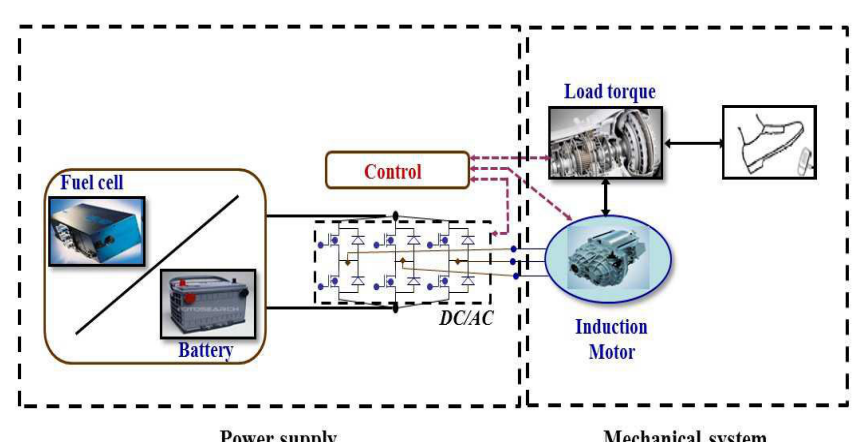

Power supply

FIG. 1: Electric vehicle powertrain

corresponds to a class of linear hybrid systems that can be written as follows:

$$
\dot{X}=f(x)+B U
$$

where:

$X=\left(I_{d s}, I_{q s}, \phi_{d r}, \phi_{q r}, \omega_{r}\right)^{T}$ is the state vector. $U=\left(U_{d s}, U_{q s}\right)^{T}$ is the control vector.

$$
\left\{\begin{array}{c}
\frac{d I_{d s}}{d t}=\frac{1}{\sigma L_{s}}\left[-\left(R_{s}+R_{r} \frac{L_{m}^{2}}{L_{r}^{2}}\right) I_{d s}+\omega_{s} L_{s} \sigma I_{q s}\right. \\
\left.+\frac{L_{m}}{T_{r} L_{r}} \phi_{d r}+p \omega_{m} \frac{L_{m}}{L_{r}} \phi_{q r}+v_{d s}\right] \\
\frac{d I_{q s}}{d t}=\frac{1}{\sigma L_{s}}\left[-\sigma L_{s} \omega_{s} I_{d s}-\left(R_{s}+R_{r} \frac{L_{m}^{2}}{L_{r}^{2}}\right) I_{q s}\right. \\
\left.-p \omega_{s} \frac{L_{m}}{L_{r}} \phi_{d r}+\frac{L_{m}}{T_{r} L_{r}} \phi_{q r}++v_{q s}\right] \\
\frac{d \phi_{d r}}{d t}=\frac{L_{m}}{T_{r}} I_{d s}-\frac{1}{T_{r}} \phi_{d r}+\omega_{g l} \phi_{q r} \\
\frac{d \phi_{q r}}{d t}=\frac{L_{m}}{T_{r}} I_{q s}-\frac{1}{T_{r}} \phi_{q r}-\omega_{g l} \phi_{d r} \\
\frac{d \omega_{m}}{d t}=\frac{1}{J}\left(C_{e m}-C_{r}+k_{f} \omega_{m}\right)
\end{array}\right.
$$

$\omega_{g l}=\omega_{s}-\omega, \omega_{s}=\frac{d \theta}{d t}, \omega=p \omega_{m}$.

$C_{e m}=p\left(I_{d s} \phi_{d r}-I_{q s} \phi_{q r}\right), \phi_{r}=\sqrt{\phi_{d r}^{2}+\phi_{q r}^{2}}$.

$\sigma=1-\frac{L_{m}}{L_{s} L_{r}}, T_{r}=\frac{L_{r}}{R_{r}}$.

where $J$ is the moment of inertia. $L_{r}, L_{s}$ and $L_{m}$ are respectively rotor inductance, stator inductance and mutual inductance. $R_{r}$ and $R_{s}$ are respectively the resistance of the rotor and of the stator, $p$ is the number of poles pairs of the machine, $k_{f}$ is the friction coefficient and $\sigma$ is Blondel's dispersion coefficient. The machine parameters are given in the appendix.

\section{B. Induction Field Oriented Control}

The aim of the frequency control method (Slipway Frequency Control) consists in using the rotor flux position calculated according to the reference variables instead of its amplitude. This method does not use a flux sensor (physical sensor or dynamic model) but needs the rotor speed sensor [?]. Fig.?? shows the structure of the applied indirect Field Oriented Control (FOC) with a $P I$ or $H_{\infty}$ controller for the speed regulation of the induction motor fed by three level inverter controlled by a $P W M$ strategy.

The FOC (Field Oriented Control) is an arithmetic block which has two inputs $\left(\phi_{r}^{*}, C_{e m}^{*}\right)$ and generates five reference variables $\left(V_{d s}^{*}, V_{q s}^{*}, \omega_{s}, I_{d s}^{*}, I_{q s}^{*}\right)$. It is based on the steady state for which the rotor flux and the electromagnetic torque are maintained constant and equal to their reference values. By neglecting the dynamics of the direct current and the torque current components, the equations of this block are given by the following expressions:

$$
\left\{\begin{array}{c}
I_{d s}^{*}=\frac{\phi_{r}^{*}}{L_{m}} \\
I_{q s}^{*}=\frac{L_{r} C_{e m}}{p L_{m} \phi_{r}^{*}} \\
\omega_{s}^{*}=\omega_{m}+\frac{L_{m} R_{r} I_{q s}^{*}}{L_{r} \phi_{r}^{*}} \\
V_{d s}^{*}=R_{s} I_{d s}^{*}-\omega_{s}^{*} \sigma L_{s} I_{q s}^{*} \\
V_{q s}^{*}=R_{s} I_{q s}^{*}-\omega_{s}^{*} \sigma L_{s} I_{d s}^{*}
\end{array}\right.
$$

This method consists in controlling the direct component $I_{d s}$ and the torque current component $I_{q s}$ in order to obtain the desired electromagnetic torque and flux in the machine.

\section{FTC CONTROLlER ARCHITECTURE}

In this section, we use the FTC architecture displayed in Fig.?? in order to ensure both performance and robustness . Firstly, we consider the feedback diagram presented in Fig.??, in which the signal of the fault detection FDI $r$ controls the switching between the two controllers.

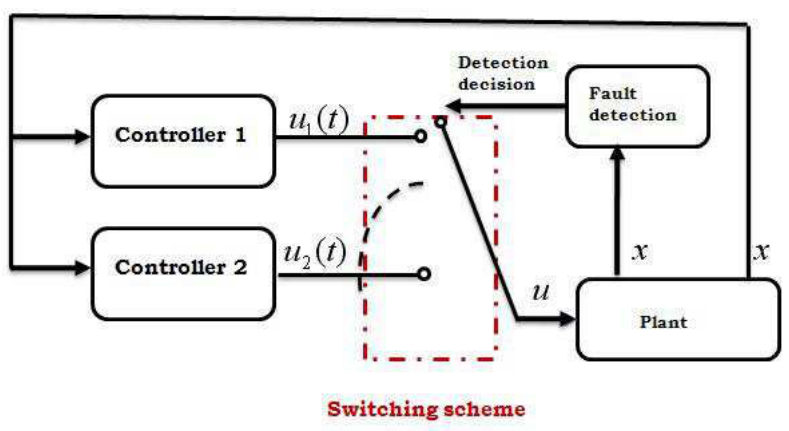

FIG. 2: Hybrid FTC structure

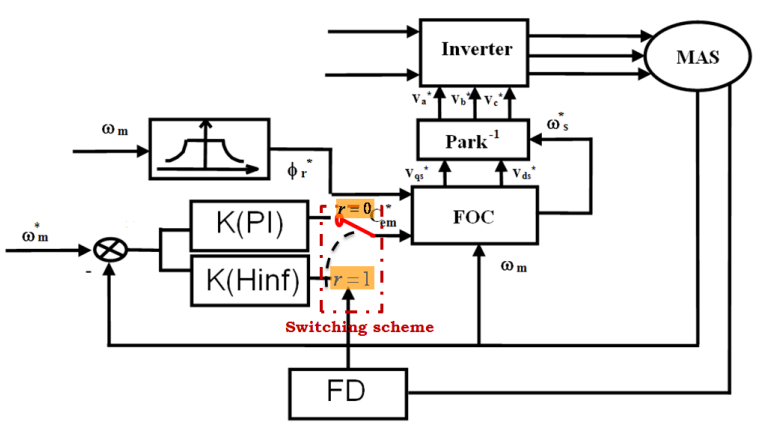

FIG. 3: FTC structure with FOC control for induction motor

In healthy case (no fault), $r=0$, we use a $P I$ controller. At fault occurence (model uncertainty or other sources of 
uncertainties such as disturbances and sensor noises), $r=$ 1 , the $H_{\infty}$ loop control is activated. Thus, $K($ hinf $)$ can be designed to robustify the system. It follows that the controller design architecture has a clear separation between performance and robustness.

In the fault diagnosis literature, $r$ is used to detect the possible faults in actuator and/or sensors. $r=0$ signifies nominal system without any failure. If $r=1$ a robust controller is implemented using the structure shown in Fig.??. The fault tolerant controllers can be designed such that they provide adequate performance when there are no faults in the systems and as much tolerance as possible using a robust controller in the presence of the faults.

The signal $r$ is computed as the residual between the actual speed and the speed estimated with an Extended Kalman Filter (EKF) [?]. Therefore $r=\omega-\hat{\omega}$, and the decision is made as:

$$
\begin{array}{lll}
r=1 & \text { if } & \omega-\hat{\omega} \neq 0 \\
r=0 & \text { if } & \omega-\hat{\omega}=0
\end{array}
$$

In this structure, the $K$ controller is implemented with a switching system which is activated by the residual $r$. If the system model with its parameters are not perfectly identified, which is the most common case, so the FDI output $r$ will be already different of zero. In order to increase the robustness of the switching, we choose to impose the following condition.

$$
\begin{array}{ccc}
K=K(P I) & \text { if } & r=0 \\
K=K\left(H_{\infty}\right) & \text { if } & r=1
\end{array}
$$

\section{A. $H_{\infty}$ Coprime Factorization Approach}

In this section, we describe the design of controller $K$ to obtain a reasonable level of robustness.

An approach was developed by Mc.Farlane and Glover [?] [?] starting from the concept of the coprime factorization of transfer matrix. This approach presents interesting properties and its implementation is based on traditional control notions.

\section{B. Robust Controller Design using Normalized Coprime Factor}

where $\widetilde{G}$ is a left coprime factorization (LCF) of $G$, and $\Delta_{M}, \Delta_{N}$ are unknown and stable transfer functions representing the uncertainty. We can then define a family of models as follows :

$$
\xi_{\epsilon}=\left\{\widetilde{G}=\left(\widetilde{M}+\Delta_{M}\right)^{-1}\left(\widetilde{N}+\Delta_{N}\right):\left\|\left(\Delta_{M} \Delta_{N}\right)\right\|_{\infty}<\epsilon_{\max }\right\}
$$

where $\epsilon$ represents the margin of maximum stability. The robust stability problem is thus to find the greatest value of $\epsilon=\epsilon_{\max }$, so that all the models belonging to $\xi_{\epsilon}$ can be stabilized by the same controller $K$. The problem of robust stability $H_{\infty}$ amounts finding and $K(s)$ stabilizing $G(s)$ so that :

$$
\left\|\left(\begin{array}{c}
I \\
K
\end{array}\right) \cdot(I-K . G)^{-1}(I G)\right\|=\gamma_{\min }=\frac{1}{\epsilon_{\max }}
$$

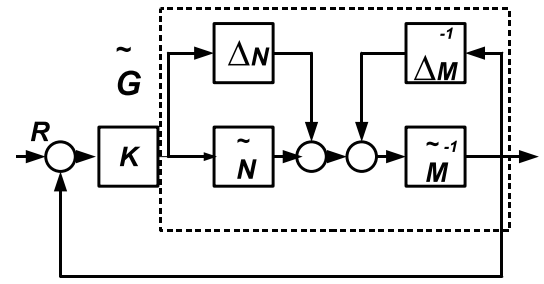

FIG. 4: Coprime factor robust stablization problem.

We define the nominal model of the system to be controlled from the coprime factors on the left: $G=\widehat{M^{-1}} \widetilde{N}$. Then a perturbed model is written (see Figure (??)):

$$
\widetilde{G}=\left(\widetilde{M}+\Delta_{M}\right)^{-1}\left(\widetilde{N}+\Delta_{N}\right)
$$

However, Mc Farlane and Glover [?] showed that the minimal value of $\gamma$ is given by :

$$
\gamma_{\min }=\epsilon_{\max }^{-1}=\sqrt{1+\lambda_{\text {sup }}(X Y)}
$$

where $\lambda_{\text {sup }}$ indicates the greatest eigenvalue of $\mathrm{XY}$, moreover for any $\epsilon<\epsilon_{\max }$ a controller stabilizing all the models belonging to $\xi_{\epsilon}$ is given by :

$$
\begin{aligned}
K(s) & =B^{T} X\left(s I-A+B B^{T} X-\gamma^{2} Z Y C^{T} C\right)^{-1} \gamma^{2} Z Y C^{T} Z \\
Z & =\left(I+Y X-\gamma^{2} I\right)^{-1}
\end{aligned}
$$

where $A, B$ and $C$ are state matrices of the system defined by the function $G$, and $X, Y$ are the positive definite matrices and the solution of the Ricatti equation :

$$
\begin{aligned}
A^{T} X+X A-X B^{T} B X+C^{T} C & =0 \\
A Y+Y A^{T}-Y C^{T} C Y+B B^{T} & =0
\end{aligned}
$$

\section{The Loop-Shaping Design Procedure}

Contrary to the approach of Glover-Doyle, no weight function can be introduced into the problem. The adjustment of the performances is obtained by affecting an open modelling (loop-shaping) process before calculating the corrector. The design procedure is as follows :

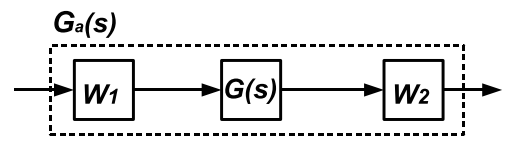

(a)

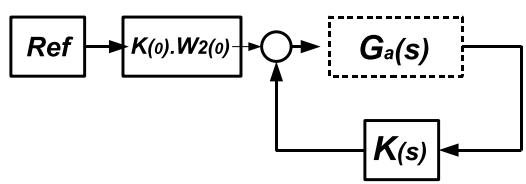

(b)

FIG. 5: The loop-shaping design procedure.

1) We add to the matrix $G(s)$ of the system to be controlled a pre-compensator $W_{1}$ and/or a postcompensator $W_{2}$, the singular values of the nominal plant are shaped to give a desired open-loop shape. The 
nominal plant $G(s)$ and shaping functions $W_{1}$ and $W_{2}$ are combined in order to improve the performances of the system so that $G_{a}=W_{1} G W_{2}$ (see Figure (??)). In the monovariable case, this step is carried out by controlling the gain and the phase of $G_{a}(j \omega)$ the Bode diagram .

2) From coprime factorizations of $G_{a}(j \omega)$, we apply the previous results to calculate $\epsilon_{\max }$, and then synthesize a stabilizing controller $K$ ensuring a value of $\epsilon$ slightly lower than $\epsilon_{\max }$ :

$$
\left\|(K)\left(I-K W_{2} G W_{1}\right)^{-1}\left(I \quad W_{2} G W_{1}\right)\right\|_{\infty}=\gamma=\frac{1}{\epsilon}
$$

3) The final feedback controller is obtained by combining the $H_{\infty}$ controller $K$ with the shaping functions $W_{1}$ and $W_{2}$ so that $K_{\infty}=W_{1} K W_{2}$.(See Figure (??))

\section{Speed Controller}

Using the indirect field oriented control for the induction motor, we have $\phi_{d r}=\phi_{r}$ and $\phi_{q r}=0$. In this case, the electromagnetic torque can be written as:

$$
C_{e m}=K I_{d s} I_{q s}, \quad K=\frac{P}{L_{r}}
$$

The induction motor model is therefore simplified and becomes:

$$
\left\{\begin{array}{c}
\frac{d I_{d s}}{d t}=\frac{1}{\sigma L_{s}}\left[-\left(R_{s}+R_{r} \frac{L_{m}^{2}}{L_{r}^{2}}\right) I_{d s}+\omega_{s} L_{s} \sigma I_{q s}\right. \\
\left.\quad+\frac{L_{m}}{T_{r} L_{r}} \phi_{r}+v_{d s}\right] \\
\frac{d I_{q s}}{d t}=\frac{1}{\sigma L_{s}}\left[-\sigma L_{s} \omega_{s} I_{d s}-\left(R_{s}+R_{r} \frac{L_{m}^{2}}{L_{r}^{2}}\right) I_{q s}\right. \\
\left.-\omega_{m} \frac{L_{m}}{L_{r}} \phi_{r}++v_{q s}\right] \\
\frac{d \phi_{r}}{d t}=\frac{L_{m}}{T_{r}} I_{d s}-\frac{1}{T_{r}} \phi_{r} \\
\frac{d \omega_{m}}{d t}=\frac{p^{2} L_{m}}{L_{r} J} I_{q s} \phi_{r}-\frac{f}{J} \omega_{m}-\frac{p}{J} C_{r} \\
\omega_{s}=\omega_{m}+\frac{L_{m} R_{r}}{L_{r} \phi_{r}} I_{q s}
\end{array}\right.
$$

By using Laplace transform, we can write:

$$
\left\{\begin{array}{c}
\phi_{r}=\frac{L_{m}}{1+\frac{L_{r}}{L_{s}} s} I_{d s} \\
C_{e m}=\frac{P L_{m}}{L_{r}} \phi_{r} \\
\omega_{m}=\frac{C_{e m}}{J s+k_{f}}
\end{array}\right.
$$

The equation (??) shows that we can control independently the rotor flux and the electromagnetic torque respectively by $I_{d s}$ and $I_{q s}$. We can represent our system by combining equations (??) and (??) in two sub-systems with the following transfer functions:

$$
G_{\phi}=\frac{1 / T_{r}}{S+1 / T_{r}}, \quad G_{\omega_{m}}=\frac{1 / J}{S+k_{f} / J}
$$

The synthesis of the FTC speed controller $K(s)$ is obtained according to the implementation showed in Fig.??. For the ideal model, the controller is chosen so as to minimize the static error. Thus, a $P I$ is chosen, $K_{s}(p)=K_{c}\left(\left(1+\tau_{c} p\right) / \tau_{c} p\right), K_{c}, \tau_{c}$ are computed in order to ensure a good margin of stability. The $H_{\infty}$ controller $K($ hinf $)$ can be designed using the loop shaping robust technique.

The fault tolerant control problem depends strongly on the type of faults that can appear in the system. In this paper, the faults is described as a multiplicative fault. In connection with FTC, this might not be very useful. The reason is that the multiplicative faults can be considered as an exponential gain drop. which will not cause any changes in the system dynamics. So the faults will not degrade the stability of the closed-loop system but the performances will be affected.

The synthesis of the $H_{\infty}$ speed controller $K(s)$ is obtained according to the implementation shown in Figure (??) using the MATLAB function ncfsyn of $\mu$-Analysis and Synthesis toolbox [?]. The controller $K(s)$ is obtained by combining the pre-filter $W_{1}$ and the post-filter $W_{2}$. Weighting functions are chosen to keep a good trade off between a relative fast time response and a good robustness [?].

$$
W_{1}=K_{c} \frac{\tau p+1}{\tau p} \quad W_{2}=\frac{k}{p^{2}+2 \xi w_{n} p+w_{n}^{2}}
$$

where: $K_{c}=0.04, \quad \tau=0.01 s, \quad k=10^{4}, \quad \xi=$ $0.707, w_{n}=100 \mathrm{rad} / \mathrm{s}$.

\section{E. Stability of Hybrid FTC Control}

For the stability of the hybrid system given by the figure Fig.??, we must search the common quadratic Lyapunov function $(C Q L F), x^{T} P x$. Where the matrix $P$ ensures the following conditions [?]:

$$
\begin{aligned}
P & >0 \\
A_{1}^{T} P+P A_{1} & <0 \\
A_{2}^{T} P+P A_{2} & <0
\end{aligned}
$$

A necessary condition for the existence of a $C Q L F$ is that the matrices $\alpha A_{1}+(1-\alpha) A_{2}$ and $\alpha A_{1}+(1-\alpha) A_{2}^{-1}$ must be Hurwitz for $\alpha \in[0,1]$. We find the matrices $A_{1}$ and $A_{2}$ by the space state representation of the closed loop transfer function respectively with $P I$ and $H_{\infty}$ controller. With these matrices $A_{1}$ and $A_{2}$ we have found the matrix $P$ guarantees the exponential stability of our hybrid system. The matrices $A_{1}, A_{2}$ and $P$ are given in the appendix.

\section{Simulation Results}

In this section, the fault tolerant controller is evaluated, based on the structure shown in the block diagram of Fig.??. The FDI output signal $r$ as it can be seen in Fig.?? activates the controller which output will be applied to the Field Control Block.

The simulation results given in Fig.?? show in the healthy case the speed and flux responses with good performances with the $P I$ controller.

For the $H_{\infty}$ controller design, the specifications are 

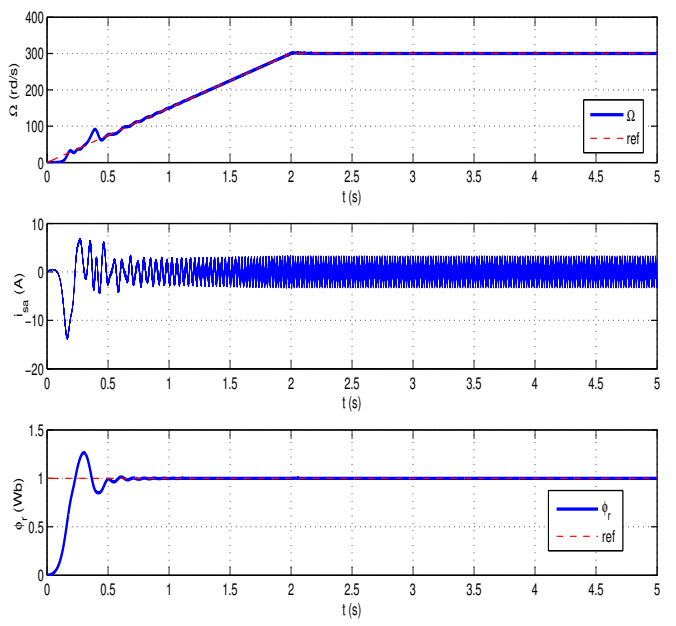

FIG. 6: Response of the induction motor without a fault sensor using $P I$ controller.

taken to ensure : (a) the speed should track the reference speed. (b) the control $U$ should not exceed a pre-specified saturation limit and (c) the rejection of the fault. It ensures $\|f(G, K)\|_{\infty}<\gamma$ for all $\|\Delta\|_{\infty}<\epsilon_{\max }$.

\section{A. Hybrid FTC Simulation Results}

For FTC architecture, the system has been simulated with a speed sensor fault occurence at $t=6 \mathrm{sec}$. The fault type is an exponential fault [?], it emulates a progressive bias on the actual speed measurement. The speed sensor output is then given by the following expression:

$$
\hat{\omega_{m}}=\left\{\begin{array}{l}
\omega_{m} \quad t<t_{\text {on }} ; \\
\omega_{m}\left[1-(1 / 3)\left(1-e^{-15\left(t-t_{\text {on }}\right.}\right)\right) \quad t \geq t_{\text {on }} .
\end{array}\right.
$$

Where $t_{o n}=6 s$ is the activation time of the fault, $\hat{\omega}_{m}$ the angular velocity measured by the sensor, and $\omega_{m}$ its real value.

The results of the system with a faulty speed sensor are shown in Fig.??. As it can be seen the system is stable with good performances before fault occurrence at $6 \mathrm{~s}$ but it is also clear that the $P I$ controller can't maintain the performances in case of a faulty sensor as illustrated by the motor speed, current and flux waveforms respectively in Fig. ??. Consequently the drive behavior is unacceptable and a backup strategy must be engaged to preserve the security of the vehicle.

Before fault occurrence, the residual (error between the real and estimated speed) is about $2 \%$ as it can be seen in Fig.??.a. In normal operation, the FDI signal as shown in Fig.??.b is always less than the threshold which has been determined as a multiple of the standard deviation in normal operation. At fault occurrence, the residual increases and the FDI signal exceeds the threshold value and activates the switching between the $P I$ controller and the robust controller; as it can be seen in Fig.??.c.
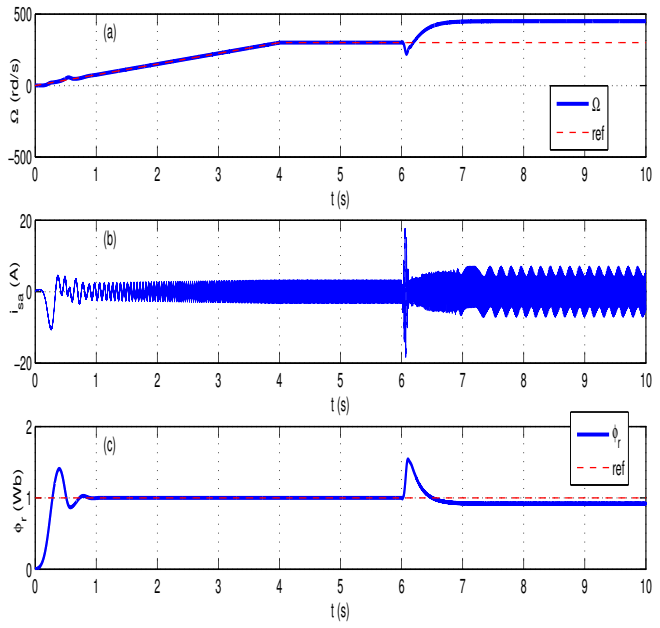

FIG. 7: Results of the induction motor drive with a faulty speed sensor.
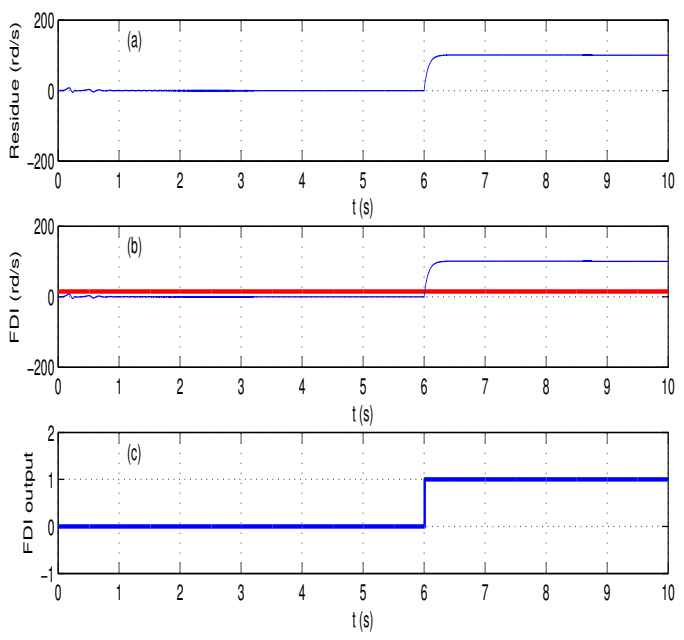

FIG. 8: FDI simulation results with exponential fault type at $6 s$.

Based on the design of $K$ (see Fig.??), the response of the induction motor drive is shown in Fig.?? where it should be noticed that the loop shaping robust controller is independent of the nominal $P I$ controller. In the worst case, our controller implementation will be equivalent to the existing robust control design. Therefore similar results of robustness and fault tolerance should be obtained in the presence of other faults like additional noise or/and in the presence of disturbances.

By observing the results displayed in Fig.??, we note that despite small undesirable effects during the transition after fault detection, the fault is perfectly handled by the robust controller. The transient effect is due to the FDI time delay and the abrupt switching. 

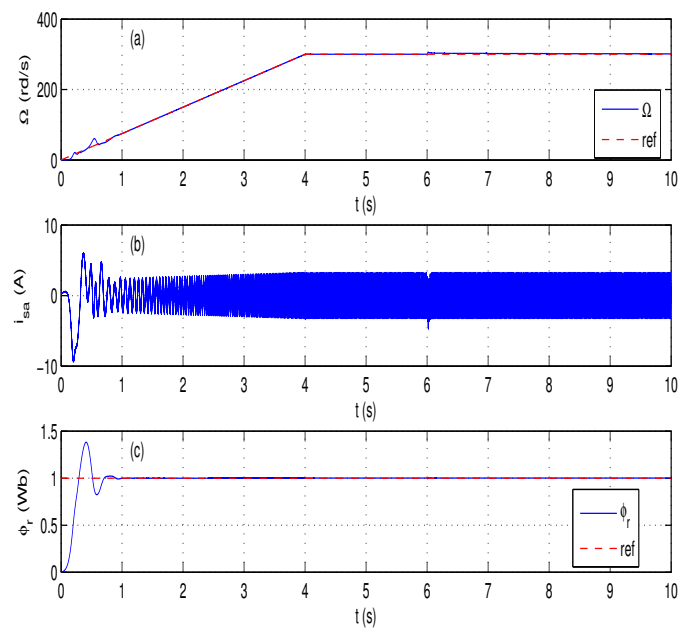

FIG. 9: Results of the induction motor drive using hybrid FTC controller at $6 s$.
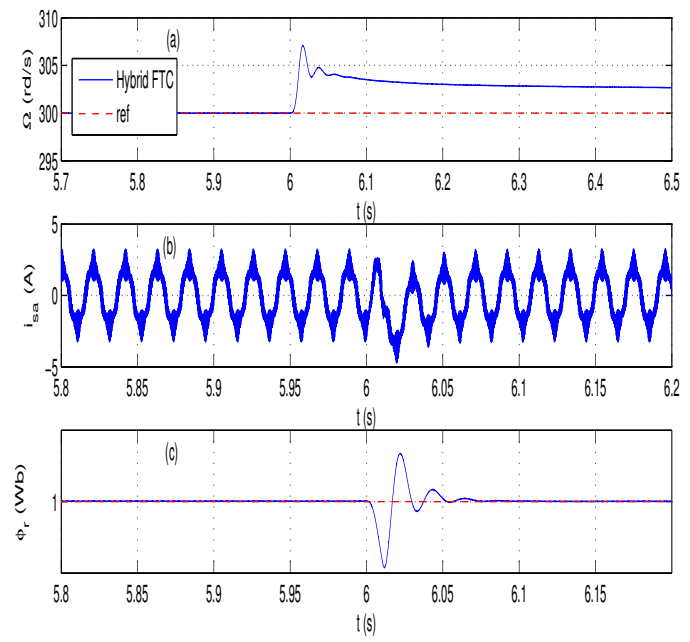

FIG. 10: Zoom around fault appearance at $\mathrm{t}=6 \mathrm{~s}$.

\section{CONCLUSION}

In this paper, an architecture of a hybrid fault tolerant control has been designed and applied to the speed control of an induction motor drive dedicated to EV. An additional controller parameter has been introduced as the main tool to achieve fault tolerance. A feature of this structure is that it automatically includes a diagnostic signal of the speed sensor. The simulation results show that the proposed method provides adequate performances when there is no fault in the system and provides resilience by using a $H_{\infty}$ robust controller. In the future, other faults will be included in the FTC design and experimental investigation will be conducted with an EV environment.

\section{REFERENCES}

[1] Wang, R.; Wang, J., "Fault-Tolerant Control with Active Fault Diagnosis for Four-Wheel Independently-Driven Electric Ground Vehicles", IEEE Transactions on Vehicular Technology, , Vol. 60, Issue 09, Page(s): 4276-4287, Nov 2011.

[2] Bennett, J.; Atkinson, G.; Mecrow, B.; Atkinson, D.; J. Bennett , "Fault Tolerant Design Considerations and Control Strategies for Aerospace Drives ”, IEEE Transactions on Industrial Electronics, Vol. 59, Issue 05, Page(s): 2049-2058, May 2012.

[3] Kang, Y.; Li, Z.; Dong, Y.; Xi, H., ' Markovian-Based Fault-Tolerant Control for Wheeled Mobile Manipulators ", IEEE Transactions on Control Systems, Vol. 20, Issue 01, Page: 266-276, Jan 2012.

[4] Betin, F.; Capolino, G., "Shaft Positioning for Six-Phase Induction Machines with Open Phases Using Variable Structure Control", IEEE Transactions on Industrial Electronics, Vol. 59, Issue 06, Page(s): 26122620, June 2012.

[5] H.C. Liaw, P. Shirinzadeh, J. Smith, "Displacement Sensor Fault Tolerance for Hydraulic Servo Axis", International Federation of Automatic Control World Conference, July 6-11,2008.

[6] M.Oudghiri-Bentaie, M. Chadli and A. El hajjaji, "Control and Sensor Fault-Tolerance of Vehicle Lateral Dynamics", International Federation of Automatic Control World Conference, July 6-11, 2008.

[7] Xiao, B.; Hu, Q.; Zhang, Y., " Adaptive Sliding Mode Fault Tolerant Attitude Tracking Control for Flexible Spacecraft Under Actuator Saturation ", IEEE Transactions on Control Systems , Vol. 20, Issue 06, Page(s): 1605-1612, Nov 2012.

[8] Yimin Gao; Ehsani, M, "Design and Control Methodology of Plug-in Hybrid Electric Vehicles ", IEEE Transactions on Industrial Electronics, Vol. 57, Issue 09, Feb 2010.

[9] Laskaris, K.I.; Kladas, A.G.,"Internal Permanent Magnet Motor Design for Electric Vehicle Drive ”, IEEE Transactions on Industrial Electronics, Vol. 57, Issue 01, Jan 2010.

[10] M. Boukhnifer, A. Chaibet and C. Larouci, "Robust Shaping Indirect Field Oriented Control for Induction Motor", Chapter Intech Books, ISBN 978-953-307-041-4, Mars 2010

[11] F. Auger, M. Hilairet; 'Industrial Applications of the Kalman Filter: A Review, 'IEEE Transactions on Industrial Electronics, 2013.

[12] D. McFarlane, K. Glover, " An $H_{\infty}$ design procedure using robust stabilization of normalized coprime factors ", IEEE Conference on Decision and Control, Austin, TX,1988.

[13] D. McFarlane, K. Glover, " Robust controller design using normalised coprime factor plant descriptions ", lecture Notes in Control and information Sciences, Vol. 138, New York: Springer Verlag, 1989.

[14] G. J. Balas, J. C. Doyle, K. Glover, A. Packard, and R. Smith, $\mu$ Analysis and Synthesis Toolbox. Natick, MA: The MathWorks, Inc, 1994.

[15] S. Skogestad and I. Postlethwaite, Multivariable Feedback Control: Analysis and Design. New York, USA: Jhon Wiley Sons, 1996/

[16] D. Liberzon, Switching in systems and control, Boston, MA Birkhauser, 2003.

[17] C. Delgado, S. Martinez-Martinez and K. Zhou, "Integrated Faultolerant scheme for a DC Speed Drive", IEEE/ASME Transaction on Mechatronics, Vol. 10, No. 4, Page(s): 419 - 427, August 2005.

\section{APPENDIX}

Parameters and Constants:

$R_{s}=0.68 \Omega, R_{r}=0.39 \Omega, L_{s}=0.156 H, L_{r}=0.156 H, M=0.22 H$ $J=0.01 \mathrm{~kg} . \mathrm{m}, k_{f}=0.001 \mathrm{IS}$

Rated data of the simulated induction motor:

$7.5 \mathrm{~kW}, 50 \mathrm{~Hz}, 230 / 380 \mathrm{~V}(\Delta / \mathrm{Y}), 23.6 / 14 A(\Delta / Y), 2900 \mathrm{rpm}$, pole pair (1), power factor (0.94).

$A_{1}=\left(\begin{array}{ccccc}-70.1 & -100 & 0 & 0 & 0 \\ 1 & 0 & 0 & 0 & 0 \\ 0 & 0 & 0 & 0 & 0 \\ 0 & 0 & 0 & 0 & 0 \\ 0 & 0 & 0 & 0 & 0\end{array}\right), A_{2}=$

$\left(\begin{array}{cccccc}-196 & -1.77 .10^{-4} & -6.51 .10^{-5} & -1.12 .10^{-7} & -7.95 .10^{-7} \\ 1 & 0 & 0 & 0 & 0 \\ 0 & 1 & & 0 & 0 & 0 \\ 0 & 0 & & 1 & 0 & 0 \\ 0 & 0 & & 0 & 1 & 0 \\ 0.15 & -1.67 & 2.47 & -7.98 & 5.17 \\ -1.67 & 2.71 & -2.97 & 5.82 & -1.06 \\ 2.47 & -2.97 & 5.50 & -6.05 & 2.15 \\ -7.98 & 5.82 & -6.05 & 0.66 & -0.59 \\ 5.17 & -1.06 & 2.51 & 0.59 & 118.05\end{array}\right)$, 